\title{
MODAL INTERACTIONS IN THE NON-LINEAR RESPONSE OF ELASTIC CABLES UNDER PARAMETRIC/EXTERNAL EXCITATION
}

\author{
N. C. Perkins \\ Department of Mechanical Engineering and Applied Mechanics. The University of Michigan, \\ Ann Arbor, MI 48109.2125 , U.S.A.
}

(Received 22 October 1990)

\begin{abstract}
A theoretical model is derived which describes the non-linear response of a suspended elastic cable to small tangential oscillations of one support. The support oscillations, in general, result in parametric excitation of out-of-plane motion and simultaneous parametric and external excitation of in-plane motion. Cubic non-lineartites due to cable stretching and quadratic nonlinearities due to equilibrium cable curvature couple these motion components in producing full, three-dimensional cable response. In this study, a two-degree-of-freedom approximation of the model is employed to examine a class of in-plane/out-of-plane motions that are coupled through the quadratic non-linearities. A first-order perturbation analysis is utilized to determine the existence and stability of the planar and non-planar periodic motions that result from simultaneous parametric and external resonances. The analysis leads to a bifurcation condition governing planar stability and results highlight how planar stability is reduced and non-planar response is enhanced whenever a "two-to-one" internal resonance condition exists between a pair of in-plane and out-of-plane cable modes. This two-to-one resonant behavior is clearly observed in experimental measurements of cable response which are also in good qualitative agreement with theoretical predictions.
\end{abstract}

\section{BACKGROUND AND INTRODUCTION}

Cables are lightweight, flexible structural elements that are capable of transmitting forces, carrying payloads and conducting signals across large distances. Due to their overall flexibility, cables are susceptible to oscillations which may ultimately degrade their performance. The study of cable dynamics has enjoyed a long and rich history [1] and recent developments in this field are reviewed in $[2,3]$. Key results of the linear theory of suspended cables, which are prerequisite to the current investigation, are briefly reviewed first.

The three-dimensional, linear response of the planar, suspended cable of Fig. 1 is the superposition of two decoupled motions: one lying in the plane defined by the cable equilibrium and one normal to this plane. The natural frequency spectrum for suspended cables with small sag and level supports is shown in Fig. 1 as a function of a cable parameter introduced by Irvine and Caughey [4]. For the symmetric suspensions considered, the in-plane vibration modes are either antisymmetric or symmetric with respect to the mid-span. Furthermore, only the symmetric modes involve (first-order) cable stretching and their frequencies depend strongly on the cable parameter. By contrast, the natural frequencies of the antisymmetric in-plane modes and all out-of-plane modes are independent of the cable parameter. The natural frequencies of in-plane modes exhibit the frequency crossover behavior shown in Fig. 1. As discussed in [4], the frequency crossovers reflect the transition of each symmetric in-plane cable mode from that of a taut string $(\lambda / \pi \rightarrow 0)$ to that of an inextensible cable $(\lambda / \pi \rightarrow \infty)$.

Hagedorn and Schafer [5] demonstrate that geometrical non-linearities significantly influence the non-linear frequencies for free, in-plane cable oscillations approximated by a single-degree-of-freedom model. In such models, both hardening and softening behaviors may occur depending on the relative contributions of the quadratic and cubic nonlinearities as determined by the cable parameter and the amplitude of oscillation $[6,7]$. The quadratic non-linearities produce a static "drift" towards the center of curvature and result in planar oscillations that are not symmetric about the equilibrium position [7]. In general, 


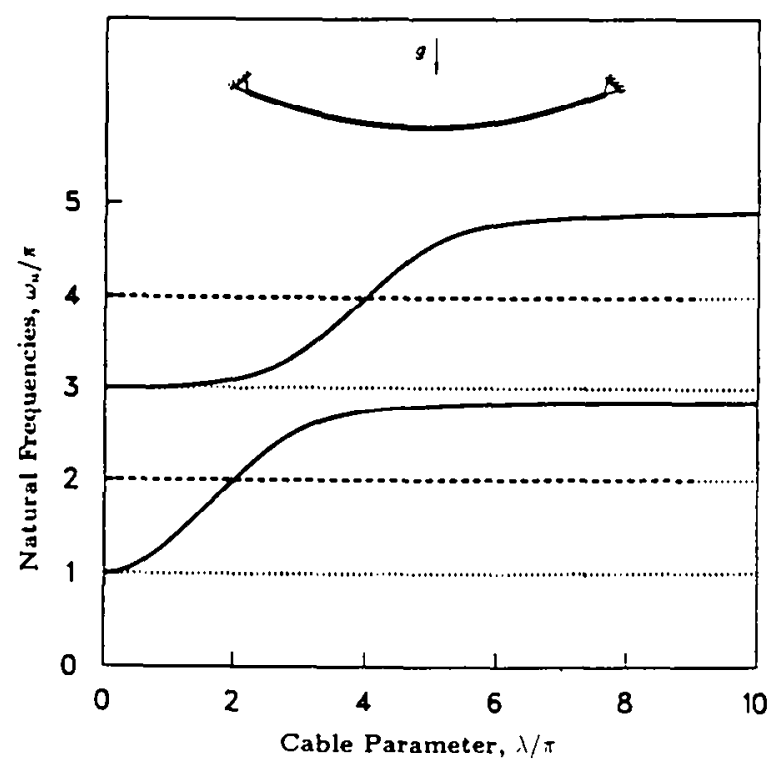

Fig. 1. Natural frequency spectrum for elastic cable with small sag and level supports. Natural irequencies of first four in-plane and out-of-plane modes are plotted vs $i, \pi$ where $\lambda^{2}$ is the cable parameter introduced in [4]. (-) symmetric in-plane modes; $(\ldots)$ antisymmetric in-plane modes; $(\cdots)$ out-of-plane modes. The $n$th crossover region is centered about $\lambda^{\prime} \pi=2 n$.

the non-linearities couple the in-plane and out-of-plane motions leading to free, threedimensional response. Luongo et al. [8] utilize a simplified two-degree-of-freedom model for transverse in-plane and out-of-plane motions to demonstrate the existence of planar and non-planar monofrequent oscillations. Monofrequent oscillations, however, arise only for particular initial conditions and, in general, modal coupling leads to a beating-type exchange of energy between in-plane and out-of-plane modes [9]. This coupling is enhanced by internal resonance between the in-plane and out-of-plane modes [10]. Qualitatively similar conclusions hold for cables with arbitrary sag and either horizontal or inclined supports [11].

Benedettini and Rega [12] examine the existence and stability of planar, periodic motions under conditions of primary external resonance. For suspensions with very small sag, the dominant non-linearities are cubic and hardening and lead to one unstable and two stable periodic solutions near resonance. For larger sag, quadratic non-linearities dominate, and up to five periodic solutions may exist close to primary resonance. Subsequent analyses focus on secondary external resonances resulting in second- and third-order superharmonics [13] and one-half- and one-third-order subharmonics [14]. Periodic planar and non-planar motions are examined in [15] under conditions of primary in-plane and outof-plane external resonance. Takahashi and Konishi [16] demonstrate that pure in-plane excitation may initiate the large out-of-plane response observed experimentally in [17]. In [16], the out-of-plane response problem is linearized about the periodic in-plane motion and cast in the form of coupled Hill's equations from which regions of instability are numerically determined.

The above investigations of non-linear cable dynamics, with the possible exception of [16], are largely unsubstantiated by experimental resuits. The present study is motivated by laboratory measurements of non-linear cable response excited by very small tangential oscillations of one support. The support oscillations lead to parametric excitation of out-of-plane and antisymmetric in-plane modes, and simultaneous parametric and external excitation of symmetric in-plane modes. For the technically important class of cables considered in the tests, the cable parameter was in the neighborhood of the first crossover in Fig. 1. As a result, the natural frequency of the fundamental symmetric in-plane mode was nearly twice that of the fundamental out-of-plane mode. The support motion frequency was selected to excite principal parametric resonance of the fundamental out-of-plane mode, and as a consequence of internal tuning, this excitation could simultaneously excite primary 

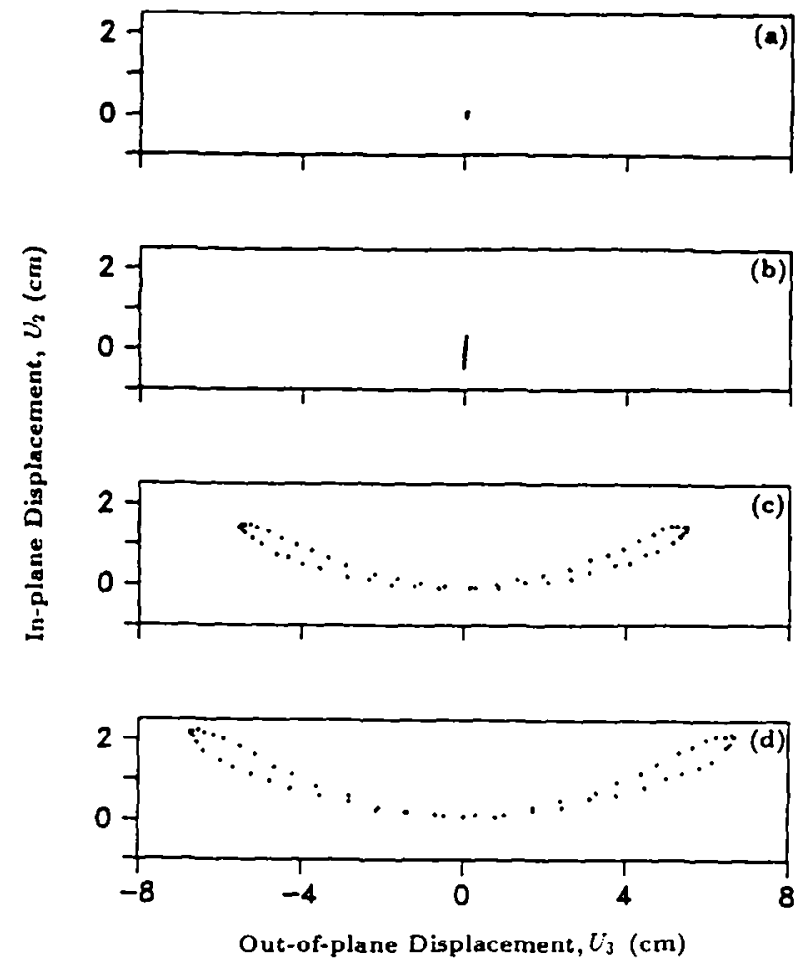

Fig. 2. Measured cable response induced by small oscillations of one support. Periodic orbits described by the cable cross-section are plotted in the normal $U_{2} \times U_{3}$ plane; refer to Fig. 9 . The sequence (a)-(d) shows the periodic orbits generated by slowly increasing the excitation amplitude $F$. (a) $F=0.12 \mathrm{~mm} \quad\left(f=3.2 \times 10^{-5}\right) ; \quad$ (b) $F=0.43 \mathrm{~mm} \quad\left(f=11.2 \times 10^{-3}\right)$; (c) $F=0.50 \mathrm{~mm}\left(f=13.8 \times 10^{-3}\right) ;$ (d) $F=0.85 \mathrm{~mm}\left(f=23.5 \times 10^{-3}\right)$.

external resonance of the fundamental symmetric in-plane mode. Under these conditions, the measured cable response was either planar or highly coupled as illustrated by the test results of Fig. 2. Pure planar, periodic response was observed for small excitation (support motion) amplitude; sce, for example, Fig. $2 a$ and b. Beyond a critical excitation amplitude, the response became suddenly non-planar and consisted of large in-plane and very large out-of-plane harmonic components. Furthermore, as illustrated in Fig. $2 \mathrm{c}$ and $\mathrm{d}$, the frequencies of the in-plane and out-of-plane motions were commensurable and in the ratio of $2: 1$, respectively. As described herein, these experimental results are evidence of the strong modal interactions which couple a commensurable pair of in-plane and outof-plane modes through quadratic non-linearities.

Nayfeh and Balachandran [18] review the many previous investigations that focus on the response of internally resonant, two-degree-of-freedom systems coupled through quadratic non-linearities. These have considered the periodic, quasi-periodic and chaotic responses excited by external resonances (e.g. $[19,20]$ ) or parametric resonances (e.g. $[21,22])$. Related experimental studies have verified the saturation phenomenon [23] and the existence of various amplitude- and phase-modulated responses [24]. Within this context, the current investigation considers the existence and stability of periodic motions that arise under conditions of simultaneous external and parametric resonances. External and parametric resonances may interact strongly in single-degree-of-freedom systems as seen, for example, in [25].

This investigation begins with the derivation of a continuum model that describes the non-linear, three-dimensional response of an elastic cable to tangential oscillations of one support. An asymptotic form of this model is developed for suspensions with small equilibrium curvature. The asymptotic model is discretized using Galerkin's method and a two-degree-of-freedom approximation is used to examine coupled in-plane/out-of-plane response. Using the method of multiple scales, a first-order perturbation analysis provides four ordinary differential equations governing amplitude and phase modulations under conditions of simultaneous principal parametric resonance of the out-of-plane mode and 
primary external resonance of the (symmetric) in-plane mode. These equations are used to determine the existence and stability of steady-state periodic motions and the bifurcation condition governing planar stability. Results from a companion experimental study are presented.

\section{THEORETICAL MODEL}

A theoretical model is derived that describes the non-linear dynamic response of an elastic cable about a curved equilibrium configuration (Fig. 3). The planar equilibrium configuration describes a cable which sags between two level supports under the influence of gravity. The response considered is three-dimensional and arises from small, prescribed oscillations $F(T)=F \cos \Omega T$ of the left support in the direction tangential to the equilibrium cable. This response $\bar{U}(S, T)$ is decomposed into the three displacement components $U_{1}(S, T), U_{2}(S, T), U_{3}(S, T)$ aligned, respectively, with the local tangential $\mathrm{l}_{1}$, normal $\mathrm{I}_{2}$, and binormal $I_{3}$ unit vectors defined by the equilibrium configuration. Here, $S$ denotes the equilibrium are length coordinate measured positive from the left support $S=0$ to the right support $S=L$ and $T$ denotes time.

\subsection{Continuous model}

The equations governing three-dimensional response are derived by applying Hamilton's principle

$$
\delta\left[\int_{T_{1}}^{T_{2}}\left(\Pi_{T}-\Pi_{S}+\Pi_{W}\right) \mathrm{d} T\right]=0
$$

in which $\Pi_{r}, \Pi_{s}$ and $\Pi_{w}$ denote the cable kinetic energy, cable strain energy, and the work done by gravity, respectively.

Following $[7,26]$, the cable is modeled as a one-dimensional, homogeneous elastic continuum obeying a linear stress-strain law. The flexible cable is assumed to undergo uniform axial extensions described by the Lagrangian strain of its centerline. This finitestrain description leads to the non-linear strain-displacement relationship [26]

$$
\varepsilon=\frac{P}{E A}+U_{1, s}-K U_{2}+\frac{1}{2}\left[\left(U_{1, s}-K U_{2}\right)^{2}+\left(U_{2, s}+K U_{1}\right)^{2}+U_{3, s}^{2}\right]
$$

in which $\varepsilon$ is the Lagrangian strain of the cable in the final (dynamic) configuration and $K(S)$ and $P(S)$ are the cable curvature and tension in the equilibrium configuration. $E A$ denotes the section stiffness of the cable cross-section. Using (2), the strain energy of the cable in the final configuration becomes [26]

where

$$
\Pi_{S}=\Pi_{s}^{i}+\int_{0}^{L}\left[P \varepsilon_{d}+\frac{1}{2} E A \varepsilon_{d}^{2}\right] \mathrm{d} S
$$

$$
\varepsilon_{d}=U_{1, s}-K U_{2}+\frac{1}{2}\left[\left(U_{1, s}-K U_{2}\right)^{2}+\left(U_{2,5}+K U_{1}\right)^{2}+U_{3, s}^{2}\right]
$$

is the dynamic component of the Lagrangian strain and $\Pi_{s}^{i}$ is the strain energy of the equilibrium cable. The kinetic energy of the cable is

$$
\Pi_{T}=\int_{0}^{L} \frac{1}{2} \rho\left[\left(U_{1, T}\right)^{2}+\left(U_{2, \tau}\right)^{2}+\left(U_{3, \tau}\right)^{2}\right] \mathrm{d} S
$$

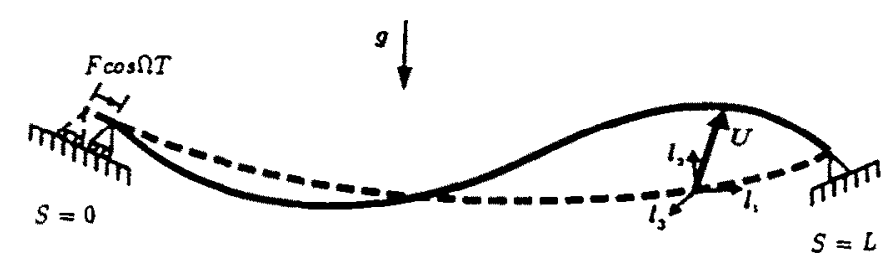

Fig. 3. Definition diagram. Elastic cable of length $L$ suspended between a fixed support $(S=L)$ and an oscillating support $(S=0) .(-$.$) equilibrium configuration; (-)$ dynamic configuration. $\mathrm{C}$ denotes three-dimensional displacement from equilibrium and is referred to the Frenet triad $\left(I_{1}, 1_{2}, l_{3}\right)$ 
where $\rho$ is the cable mass/length. The work done by gravity $g$ is

$$
\Pi_{W}=\Pi_{W}^{i}+\int_{0}^{L}-\rho g\left[U_{1} \sin \theta+U_{2} \cos \theta\right] \mathrm{d} S
$$

where $\Pi_{w}^{i}$ is the gravitational potential energy of the equilibrium cable and $\theta$ is the angle of inclination of the unit tangent vector $l_{1}$ from the horizontal.

Substitution of equations (2)-(6) into Hamilton's principal (1), and integration by parts where needed, leads to the following non-linear equations of motion:

Tangential component $U_{1}$ :

$$
\begin{aligned}
& {\left[\left(P+E A \varepsilon_{d}\right)\left(1+U_{1 . s}-K U_{2}\right)\right] . s-\left[\left(P+E A \varepsilon_{d}\right) K\left(U_{2, s}+K U_{1}\right)\right]} \\
& \quad-\rho g \sin \theta=\rho U_{1, T T} .
\end{aligned}
$$

Normal component $U_{2}$ :

$$
\begin{aligned}
& {\left[\left(P+E A \varepsilon_{d}\right)\left(U_{2, s}+K U_{1}\right)\right], s+\left[\left(P+E A \varepsilon_{d}\right) K\left(1+U_{1, s}-K U_{2}\right)\right]} \\
& \quad-\rho g \cos \theta=\rho U_{2, T r} .
\end{aligned}
$$

Binormal component $U_{3}$ :

$$
\left[\left(P+E A \varepsilon_{d}\right)\left(U_{3, s}\right)\right]_{, s}=\rho U_{3, T T} .
$$

And the boundary conditions:

$$
\begin{aligned}
& U_{1}(0, T)=F \cos \Omega T, \quad U_{1}(L, T)=0 \\
& U_{v}(0, T)=U_{v}(L, T)=0, \quad v=2,3 .
\end{aligned}
$$

Equations (7)-(9) contain both quadratic and cubic non-linearities which derive from the stretching of the cable centerline. For planar response, the quadratic non-linearities capture the softening of the cable element as it deffects towards its center of curvature. For non-planar response, the quadratic non-linearities include key terms which couple in-plane and out-of-plane motions. In the limit of vanishing equilibrium curvature, the quadratic non-linearities also vanish and equations (7)-(9) describe the non-linear response of a taut string.

The non-homogeneous terms appearing in equations (7)-(9) vanish under conditions of static equilibrium and lead to the following solution for the equilibrium tension and curvature [26]:

$$
\begin{aligned}
P(S) & =\sqrt{P_{0}^{2}+[\rho g(S-L / 2)]^{2}} \\
K(S) & =\frac{\rho g P_{0}}{P_{0}^{2}+[\rho g(S-L / 2)]^{2}}
\end{aligned}
$$

where $P_{0}$ is the equilibrium cable tension at the mid-span $(S=L / 2)$. These solutions, which describe the classical catenary cable of elementary statics, appear as non-constant coefficients in the homogeneous equations of motion reduced from equations (7)-(9).

\subsection{Asymptotic model for small curvature}

In most applications, the cable supports substantial static tension $\left(P_{0} \geq \rho g L\right)$ and the resulting equilibrium curvature is quite small. Under such conditions, the parabolic approximation to the catenary* is used which corresponds to the second-order Taylor series expansions of equations (12) and (13) about the mid-span:

$$
P=P_{0}, \quad K=\frac{\rho g}{P_{0}} .
$$

The non-dimensional curvature $k=K L=\rho g L / P_{0}$ is then identified as a small parameter and is used to order the terms in the equations of motion (7)-(9) in obtaining an asymptotic

- This approximation is valid whenever $K \leq 1 / L$ or, equivalently, whenever $D / H \leq 1 / 8$ where $D$ is the sag of the equilibrium cable at the mid-span and $H$ is the horizontal distance between the supports [1]. 
model for small curvature. Using the equilibrium solutions (12) and (13) in equations (7)-(9) and neglecting terms of order $k^{2}$ and higher provides

$$
\begin{gathered}
v_{l}^{2}\left\{u_{1, s}-k u_{2}+\frac{1}{2}\left[u_{2, s}^{2}+u_{3, s}^{2}\right]\right\}_{, s}=u_{1, n} \\
\left(\left\{v_{t}^{2}+v_{l}^{2}\left[u_{1, s}-k u_{2}+\frac{1}{2}\left(u_{2, s}^{2}+u_{3, s}^{2}\right)\right]\right\} u_{2, s}\right)_{, s}+k v_{l}^{2}\left(u_{1, s}-k u_{2}\right)=u_{2, n} \\
\left(\left\{v_{t}^{2}+v_{l}^{2}\left[u_{1, s}-k u_{2}+\frac{1}{2}\left(u_{2, s}^{2}+u_{3, s}^{2}\right)\right]\right\} u_{3, s}\right)_{, s}=u_{3, n t}
\end{gathered}
$$

where the following non-dimensional quantities have been used:

$$
\begin{aligned}
& s=\frac{S}{L}, \quad t=\frac{T}{\sqrt{L / g}}, \quad u_{i}=\frac{U_{i}}{L} \quad i=1,2,3 \\
& k=\frac{\rho g L}{P_{0}}, \quad v_{\mathrm{l}}^{2}=\frac{P_{0}}{\rho g L}=\frac{1}{k}, \quad v_{l}^{2}=\frac{E A}{\rho g L} .
\end{aligned}
$$

In equations (15)-(17), the quantities $v_{l}$ and $v_{t}$ represent the (non-dimensional) propagation speed of longitudinal waves and transverse waves along the cable, respectively.

Next, the acceleration term in equation (15) is neglected under the assumption that the cable stretches in a quasi-static manner. This assumption, discussed in detail by Irvine and Caughey for elastic cables [4], is based on the fact that the propagation speed for longitudinal waves greatly exceeds that for transverse waves and, therefore, the ratio $v_{t}^{2} / v_{l}^{2}$ is of order $k$ or smaller. Proceeding in a manner similar to [7], integration of equation (15) provides

$$
u_{1, s}-k u_{2}+\frac{1}{2}\left[u_{2, s}^{2}+u_{3, s}^{2}\right]=g(t)
$$

where $g(t)$ is an arbitrary function of time. Integrating again and using the boundary conditions (10) provides

$$
u_{1}(s, t)=f \cos \omega t+g(t) s+\int_{0}^{s}\left[k u_{2}(\eta, t)-\frac{1}{2}\left\{\left[u_{2, \eta}(\eta, t)\right]^{2}+\left[u_{3 . \eta}(\eta, t)\right]^{2}\right\}\right] \mathrm{d} \eta
$$

where

and

$$
g(t)=-f \cos \omega t+\int_{0}^{1}\left[-k u_{2}(\eta, t)+\frac{1}{2}\left\{\left[u_{2, \eta}(\eta, t)\right]^{2}+\left[u_{3, \eta}(\eta, t)\right]^{2}\right\}\right] \mathrm{d} \eta
$$

$$
f=\frac{F}{L}, \quad \omega=\Omega \sqrt{\frac{L}{g}} .
$$

Equations (20)-(22) show that, for small support oscillations of order $k$, the tangential displacement $u_{1}(s, t)$ remains of order $k$.

Substituting the above into equations (16) and (17) results in the following non-linear equations of motion governing the two transverse displacement components $u_{v}(s, t), v=2,3$

$$
\begin{gathered}
{\left[v_{t}^{2}+v_{l}^{2} g(t)\right] u_{3, s s}=u_{3, u t}} \\
{\left[v_{t}^{2}+v_{l}^{2} g(t)\right] u_{2, s s}+k v_{l}^{2} g(t)=u_{2, t}}
\end{gathered}
$$

with the boundary conditions (11)

$$
u_{v}(0, t)=u_{v}(1, t)=0 .
$$

Equations (20)-(25) constitute an asymptotic form of the three-dimensional equations of motion which is valid in the limit of (i) small equilibrium curvature, and (ii) low-frequency response. For the linear theory [4], the accuracy of the quasi-static stretching assumption deteriorates for high-frequency response involving high-order elastic modes [26]. In equations (23) and (24), the sum $\left[v_{t}^{2}+v_{l}^{2} g(t)\right]$ represents the total cable tension composed of the static component $v_{t}^{2}$ and the dynamic component $v_{t}^{2} g(t)$. The latter includes the dynamic tension induced by support motion which leads to the parametric excitation terms $-\left(v_{l}^{2} f \cos \omega t\right) u_{3, s s}$ in equation (23) and $-\left(v_{l}^{2} f \cos \omega t\right) u_{2, s s}$ in equation (24). Note also that the term $k v_{i}^{2} g(t)$ in equation (24) includes the external excitation term $-k v_{l}^{2} f \cos \omega t$. This excitation in the normal direction arises from the tangential motion of the support due to the 
(non-zero) equilibrium curvature. Finally, note that upon linearization and setting $f=0$, equations (21) and (23)-(25) reduce to the familiar linear cable theory of Irvine and Caughey [4] with their cable parameter $j^{2}=v_{l}^{2} /\left(v_{t}^{2}\right)^{3}$.

\subsection{Discrete model}

A two-degree-of-freedom model is proposed for analyzing modal interactions in coupled in-plane/out-of-plane response. The discrete model is determined from equations (21)-(25), through application of Galerkin's method using the separable solutions:

$$
\begin{aligned}
& u_{3}(s, t)=v_{3}(t) \theta_{3 j}(s) \\
& u_{2}(s, t)=v_{2}(t) \theta_{2 i}(s) .
\end{aligned}
$$

The comparison functions $\theta_{3 j}(s)$ and $\theta_{2 i}(s)$ are chosen to be the $j$ th out-of-plane and ith in-plane vibration mode shapes associated with free, linear response [4]. The natural frequencies associated with these mode shapes are denoted by $\omega_{3}$ and $\omega_{2}$, respectively. Substitution of equations (26) and (27) into equations (21), (23) and (24) and application of Galerkin's method yields the two-degree-of-freedom model:

$$
\begin{aligned}
\ddot{v}_{3}+\omega_{3}^{2} v_{3}= & -2 \hat{\mu}_{3} \dot{v}_{3}-2\left(f \hat{p}_{3} \cos \omega t\right) v_{3}+\alpha_{1} v_{2} v_{3}+\beta_{1} v_{3}^{3}+\beta_{2} v_{2}^{2} v_{3} \\
\ddot{v}_{2}+\omega_{2}^{2} v_{2}= & -2 \hat{\mu}_{2} \dot{v}_{2}-2\left(f \hat{p}_{2} \cos \omega t\right) v_{2}+f \hat{f}_{2} \cos \omega t+x_{2} v_{3}^{2} \\
& +x_{3} v_{2}^{2}+\beta_{3} v_{2} v_{3}^{2}+\beta_{4} v_{2}^{3}
\end{aligned}
$$

in which modal damping terms have been added. Note that the amplitudes of the parametric and external excitation terms are not independent and, in equations (28) and (29), their dependence on the support motion amplitude $f$ is emphasized. The coefficients appearing in equations (28) and (29) are defined in the Appendix. Also in the Appendix, it is shown that the quadratic non-linearities and the external excitation vanish $\left(x_{1}=x_{2}=\hat{f}_{2}=0\right)$ only when the in-plane mode $\theta_{2 i}(s)$ chosen in equation (27) is antisymmetric.

\section{PERTURBATION ANALYSIS}

The influence of modal interactions in coupled in-plane/out-of-plane cable response is examined through a perturbation analysis. The present analysis, which considers the coupling produced by the quadratic non-linearities in equations (28) and (29), is used to determine planar and non-planar periodic motions and their stability. The analysis highlights how the non-planar response is enhanced whenever a two-to-one internal resonance condition exists between the in-plane and out-of-plane modes. The higher-order coupling produced by the cubic non-linearities is not considered presently and is the subject of numerous related investigations of the non-linear dynamics of taut strings; see, for example, those reviewed in [27].

The method of multiple scales [27] is used to obtain a first-order approximation to equations (28) and (29) in terms of the uniform expansions

$$
v_{v}(t)=\varepsilon v_{v 1}\left(t_{0}, t_{1}\right)+\varepsilon^{2} v_{v 2}\left(t_{0}, t_{1}\right), \quad v=2,3 .
$$

Here, the small parameter $\varepsilon$ is introduced to distinguish motions with frequencies of order $\omega_{2}, \omega_{3}$ and $\omega$ occurring at the fast time scale $t_{0}=t$ from motions occurring at the slower time scale $t_{1}=\varepsilon t$. The slower time-scale motions represent small modulations of the response produced by the (quadratic) non-linearities, damping, parametric excitation, and external excitation. Thus, the small damping, parametric excitation and external excitation terms appearing in equations (28) and (29) are ordered as

$$
\hat{\mu}_{v}=\varepsilon \mu_{v}, \quad \hat{p}_{v}=\varepsilon p_{v}, \quad \text { for } v=2,3 \quad \hat{f}_{2}=\varepsilon^{2} f_{2}
$$

so that their influence is felt at the same level of approximation as the non-linearities.

Following the standard details of the multiple-scales method, one obtains the zerothorder solutions

$$
v_{v 1}=A_{v}\left(t_{1}\right) \mathrm{e}^{\mathrm{j} \omega_{v} t_{0}}+\mathrm{cc}, \quad v=2,3
$$


where the $A_{v}\left(t_{1}\right)$ are the slowly modulated response amplitudes and cc denotes the complex conjugate of the preceeding terms. The equations governing the first-order solutions are:

$$
\begin{aligned}
\frac{\partial^{2} v_{32}}{\partial t_{0}^{2}}+\omega_{3}^{2} v_{32}= & -2 i \omega_{3}\left(\frac{\mathrm{d} A_{3}}{\mathrm{~d} t_{1}}+\mu_{3} A_{3}\right) \mathrm{e}^{\left(\omega_{j}\right) t_{0}}+x_{1} A_{2} \bar{A}_{3} \mathrm{e}^{\mathrm{i}\left(\omega_{2}-\omega_{3}\right) t_{0}} \\
& -f p_{3} \bar{A}_{3} \mathrm{e}^{i\left(\omega-\omega_{3}\right) t_{0}}+\mathrm{cc}+\mathrm{nst}, \\
\frac{\partial^{2} v_{22}}{\partial t_{0}^{2}}+\omega_{2}^{2} v_{22}= & -2 i \omega_{2}\left(\frac{\mathrm{d} A_{2}}{\mathrm{~d} t_{1}}+\mu_{2} A_{2}\right) \mathrm{e}^{i \omega_{3} t_{0}}+x_{2} A_{3}^{2} \mathrm{e}^{i 2 \omega_{3} t_{0}} \\
& -f p_{2} \bar{A}_{2} \mathrm{e}^{i\left(\omega-\omega_{3}\right) t_{0}}+\frac{1}{2} f f_{2} \mathrm{e}^{i \omega t_{0}}+c c+n s t,
\end{aligned}
$$

where the overbar $\left({ }^{-}\right)$also denotes complex conjugate and nst represents terms that are always non-secular. The (non-linear) terms proportional to $x_{1}$ and $\alpha_{2}$ become secular provided (i) the in-plane mode is symmetric ${ }^{+}$and (ii) $\omega_{2} \approx 2 \omega_{3}$. These conditions arise in technical applications and are satisfied whenever the cable parameter $\lambda / \pi \approx 2 n$ where $n=1,2, \ldots$. These values of the cable parameter define the regions of frequency crossover illustrated in Fig. 2 and result in a cable described herein as tuned. The terms proportional to $p_{v}$ become secular under the conditions of principal parametric resonance: $\omega \approx 2 \omega_{v}$, $v=2$ or 3 . Furthermore, for a tuned cable, principal parametric resonance of the outof-plane mode $\left(\omega \approx 2 \omega_{3} \approx \omega_{2}\right)$ leads to primary external resonance of the in-plane mode; see secular term proportional to $f_{2}$ in equation (34). This study focuses attention on the latter case.

For the former case of principal parametric resonance of the in-plane mode, the equations (33)-(34) constitute an example of what is termed the "second form" in [18]. For this case, however, the response of a suspended cable is likely to involve more than simply two modes. Note that in the first crossover region of Fig. $1, \omega \approx 2 \omega_{2}$ implies that $(\omega / \pi \approx 4)$. Consequently, the excitation frequency is twice the natural frequencies of the first symmetric and antisymmetric in-plane modes as well as the second out-of-plane mode and all three modes would experience simultaneous principal parametric resonances. Furthermore, the parametric resonance of the first symmetric in-plane mode could drive the fundamental outof-plane mode through the two-to-one internal resonance described above. The current two-degree-of-freedom model would be insufficient to represent cable response in this case.

\subsection{Parametric resonance of out-of-plane mode}

In this case, excitation energy is imparted to the cable through principal parametric resonance of the out-of-plane mode and, simultaneously, through primary external resonance of the in-plane mode, provided the cable is nearly tuned. These conditions are described by

$$
\omega=2 \omega_{3}+\varepsilon \rho, \quad 2 \omega_{3}=\omega_{2}+\varepsilon \sigma
$$

where $\rho$ and $\sigma$ denote parametric and internal detuning parameters, respectively. Combining these definitions reveals an "external detuning parameter" given by the sum $\rho+\sigma$ which governs the external resonance of the in-plane mode. These definitions are used in equations (33) and (34) together with the polar representations $A_{v}\left(t_{1}\right)=a_{v}\left(t_{1}\right) \mathrm{e}^{\left.\mathrm{i \phi} . t_{1}\right)} / 2, v=2,3$, and the vanishing of the secular terms provides the following four equations governing the amplitude and phase modulations

$$
\begin{gathered}
\omega_{3}\left(\frac{\mathrm{d} a_{3}}{\mathrm{~d} t_{1}}+\mu_{3} a_{3}\right)-\frac{1}{4} \alpha_{1} a_{2} a_{3} \sin \gamma_{3}+\frac{1}{2} f p_{3} a_{3} \sin \gamma_{2}=0 \\
\omega_{3} a_{3} \frac{\mathrm{d} \phi_{3}}{\mathrm{~d} t_{1}}+\frac{1}{4} \alpha_{1} a_{2} a_{3} \cos \gamma_{3}-\frac{1}{2} f p_{3} a_{3} \cos \gamma_{2}=0
\end{gathered}
$$

\footnotetext{
The antisymmetric in-plane modes do not induce first-order cable stretching and the quadratic non-linearities vanish; refer to the Appendix.
} 


$$
\begin{gathered}
\omega_{2}\left(\frac{\mathrm{d} a_{2}}{\mathrm{~d} t_{1}}+\mu_{2} a_{2}\right)+\frac{1}{4} x_{2} a_{3}^{2} \sin \gamma_{3}-\frac{1}{2} \int f_{2} \sin \left(\gamma_{2}-\gamma_{3}\right)=0 \\
\omega_{2} a_{2} \frac{\mathrm{d} \phi_{2}}{\mathrm{~d} t_{1}}+\frac{1}{4} x_{2} a_{3}^{2} \cos \gamma_{3}+\frac{1}{2} f f_{2} \cos \left(\gamma_{2}-\gamma_{3}\right)=0
\end{gathered}
$$

where

$$
\gamma_{3}\left(t_{1}\right)=-2 \phi_{3}+\phi_{2}-\sigma t_{1}, \quad \gamma_{2}\left(t_{1}\right)=-2 \phi_{3}+\rho t_{1} .
$$

The singular points of equations (36)-(39) correspond to steady-state, periodic solutions and are described by the conditions $\mathrm{d} a_{v} / \mathrm{d} t_{1}=\mathrm{d} \psi_{v} / \mathrm{d} t_{1}=0, v=2,3$. Using these conditions in $(36)-(40)$ leads to

$$
\begin{gathered}
a_{3}\left(\frac{1}{2} \omega_{3} \rho+\frac{1}{4} x_{1} a_{2} \cos \gamma_{3}-\frac{1}{2} f p_{3} \cos \gamma_{2}\right)=0 \\
a_{3}\left(-\omega_{3} \mu_{3}+\frac{1}{4} x_{1} a_{2} \sin \gamma_{3}-\frac{1}{2} f p_{3} \sin \gamma_{2}\right)=0 \\
\omega_{2}(\rho+\sigma) a_{2}+\frac{1}{4} x_{2} a_{3}^{2} \cos \gamma_{3}+\frac{1}{2} f f_{2} \cos \left(\gamma_{2}-\gamma_{3}\right)=0 \\
-\omega_{2} \mu_{2} a_{2}-\frac{1}{4} x_{2} a_{3}^{2} \sin \gamma_{3}+\frac{1}{2} f f_{2} \sin \left(\gamma_{2}-\gamma_{3}\right)=0
\end{gathered}
$$

which define all singular points. Two types of solutions to equations (41)-(44) exist which are distinguished by either vanishing or non-vanishing out-of-plane motion. For vanishing out-of-plane motion, the solutions

$$
a_{3} \equiv 0, \quad a_{2}= \pm \frac{1}{2} f \frac{f_{2}}{\omega_{2} \sqrt{\mu_{2}^{2}+(\rho+\sigma)^{2}}}
$$

give the coordinate amplitudes for planar (linear) response. For non-vanishing out-of-plane motion, the solutions are determined numerically using standard Newton-Raphson iteration. This procedure yields up to two more (non-trivial) solutions, depending on the particular choice of model parameters. The stability of all periodic solutions is assessed by linearizing equations (36)-(39) about the singular points and examining the eigenvalues associated with the linearized variational equations.

Representative solutions are illustrated by the amplitude-forcing plots of Figs 4 and 5 for a typical tuned suspension described by the model parameters given in Table 1 . These figures show the amplitudes $a_{2}$ and $a_{3}$ of all periodic solutions as functions of the (nondimensional) support motion amplitude $f$. The differences observed in these figures arise from selecting different values for internal and parametric detuning. In these and all following figures, the solid (dotted) curves denote stable (unstable) periodic solutions. It should also be noted that, in all examples considered, the periodic solutions loose stability at a turning point. However, it is likely that, for other model parameters, stability may also be lost through Hopf bifurcations as noted in the special cases of pure primary external resonance or pure principal parametric resonance [18].

Shown in Fig. 4 are two examples of suspended cables with vanishing external detuning $(\rho+\sigma=0)$. For the perfectly tuned system of Fig. $4 \mathrm{a}(\rho=\sigma=0)$, the non-planar (nonlinear) solution bifurcates from the planar (linear) solution (45) at the extremely small support motion amplitude denoted by $f_{b}$. In this instance, $f_{b} \approx 0.11 \times 10^{-5}$ and the nonplanar response is readily excited for any support motion amplitudes exceeding a mere $0.00011 \%$ of the cable length. The planar solution remains stable for $f<f_{b}$ and loses stability at the bifurcation value $f=f_{b}$. For $f>f_{b}$, the planar solution is unstable and a single, stable non-planar solution exists. Note that, for the non-planar solution, the in-plane response amplitude $a_{2}$ steadily increases with increasing support motion amplitude. This feature becomes more pronounced in Fig. 5 for cases of non-vanishing external detuning. Thus, the primary parametric resonance considered here disrupts the saturation phenomenon that would otherwise occur for this model in the presence of the primary external resonance alone [18]. Figure $4 \mathrm{~b}$ shows similar behavior for the case of vanishing external detuning but non-vanishing internal and parametric detunings $(\rho=-\sigma=15)$. The non-vanishing internal and parametric detunings substantially retard the bifurcation which, in the present case, now occurs at $f_{b} \approx 1.64 \times 10^{-3}$. 


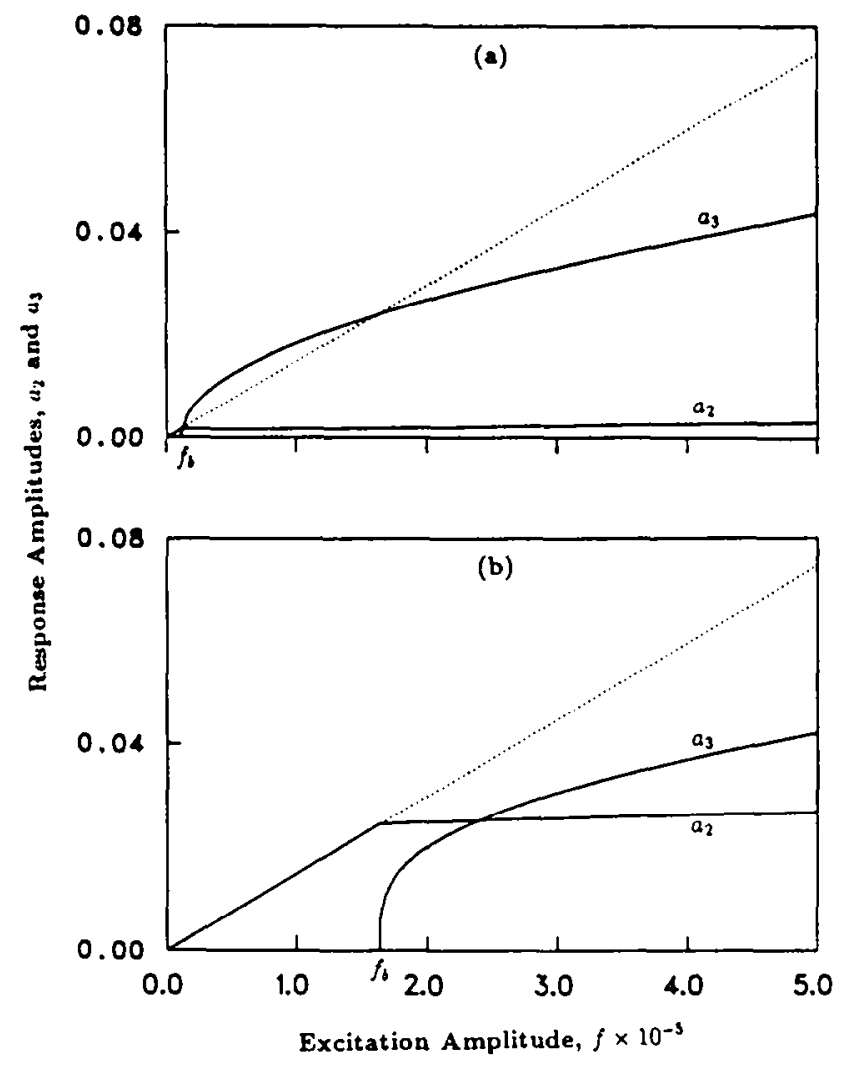

Fig. 4. Amplitude-forcing plots for cases of vanishing external detuning. Model parameters given in Table 1. (a) $\rho=\sigma=0$; (b) $\rho=-\sigma=15$. ( - ) stable solutions; $(\cdots)$ ) unstable solutions.

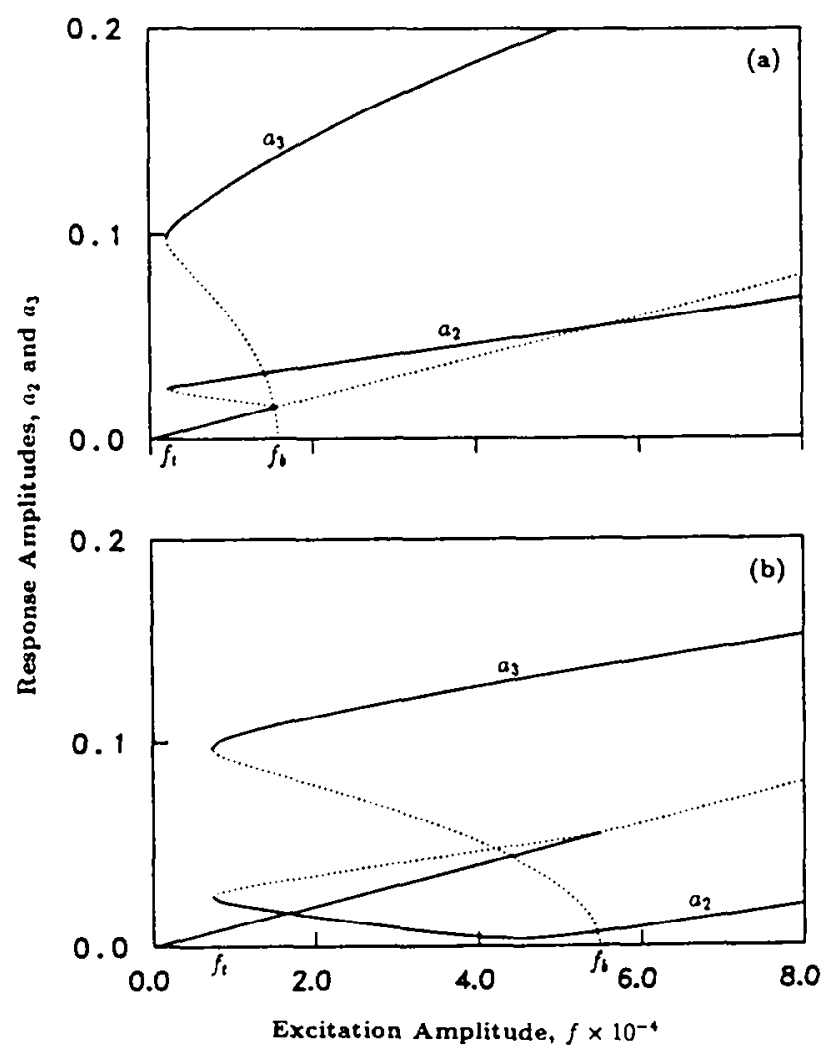

Fig. 5. Amplitude-forcing plots for cases of non-vanishing external detuning. Model parameters given in Table 1. (a) $p=\sigma-15$; (b) $\rho=\sigma--15$. (- - ) stable solutions; $(\cdots)$ ) unstable solutions. 
Table 1. Example

\begin{tabular}{ll}
\hline$i^{2}$ & 4.2 \\
$\omega_{3}$ & 6.5 \\
$\omega_{2}$ & 13 \\
$\mu_{3}$ & 0.5 \\
$\mu_{2}$ & 2 \\
$x_{1}$ & 8000 \\
$x_{2}$ & 4000 \\
$p_{3}$ & -222.000 \\
$f_{2}$ & $-78,000$ \\
\hline
\end{tabular}

Non-vanishing external detuning leads to multiple periodic solutions and jump phenomena as illustrated by the cases shown in Fig. 5 . For the case of positive external detuning $(\rho+\sigma=30)$ considered in Fig. 5a, a turning point exists at the support motion amplitude denoted by $f_{t}$ and two stable periodic solutions coexist over the range $f_{t} \leq f \leq f_{b}$. In this range, the initial conditions ultimately determine whether the cable response is planar or non-planar. Upon slowly increasing the support motion amplitude from zero, the response will remain planar while approaching $f_{0}$ from below. At $f=f_{b}$, the planar motion becomes unstable and the cable response becomes suddenly non-planar. This upward jump involves an increase in the amplitude of the in-plane response. Starting with $f>f_{b}$ and slowly decreasing $f$ leads to a downward jump in $a_{2}$ at $f=f_{t}$. These trends are reversed in the case of negative external detuning $(\rho+\sigma=-30)$ as illustrated in Fig. Sb. In particular, slowly increasing $f$ from zero in this case leads to a large decrease in $a_{2}$ at $f=f_{b}$ and, over the range shown, the resulting non-planar response is dominated by out-of-plane motion.

Figures 6 and 7 show representative amplitude-frequency plots obtained for the suspended cable example of Table 1 . The figures show the amplitudes of all periodic solutions as functions of the parametric detuning (support motion frequency). In all the cases shown, the support motion amplitude is constant $\left(f=2 \times 10^{-5}\right)$ and the observed differences arise from selecting different values for the internal detuning parameter.

In the case of vanishing internal detuning $(\sigma=0)$ considered in Fig. 6, the planar response remains stable away from the parametric and external resonances which occur, simultaneously, at $\rho=0$. Slowly increasing the support motion frequency starting from far below these resonances leads to unstable planar response at $\rho \approx-5.53$. At this detuning, the response becomes suddenly non-planar and the amplitude of the in-plane mode experiences a slight downward jump. The response remains non-planar until $\rho \approx 15.90$ where the amplitudes of both the in-plane and out-of-plane modes experience large downward jumps. When starting far above these resonances and slowly decreasing the support motion frequency, non-planar response will first occur near $\rho \approx 6.22$ and vanish below $\rho \approx-10.0$. Note that, in contrast to the case of pure external resonance [18], the additional parametric resonance considered here renders the amplitude-frequency curve asymmetric with respect to the $\rho=0$ axis for the case $\sigma=0$. From Fig. 6, also note that the non-planar response shown in Fig. 4a is driven by simultaneous parametric and external resonances.

Significant internal detuning separates the parametric and external resonances as shown by the amplitude-frequency plots of Fig. 7 . For the case of significant positive internal detuning $\sigma=15$ considered in Fig. 7a, the external resonance is centered about $\rho=-15$ and lies to the left of the parametric resonance centered about $\rho=0$. In this example, the stable response is planar in the extreme regions $\rho<-16.86$ and $\rho>13.85$ and also in the intermediate region $-12.78<\rho<-3.77$. Slowly increasing the support motion frequency starting from well below the external resonance leads to a large upward jump in $a_{3}$ and a slight downward jump in $a_{2}$ at $\rho \approx-16.03$. As $\rho \approx-12.78$ is approached from below, the amplitudes of the non-planar response components decrease rapidly and are continuous with those of the stable planar response in the intermediate region. Increasing the support motion frequency eventually produces a very rapid but continuous increase in $a_{3}$ and a modest and continuous decrease in $a_{2}$ near $\rho \approx-3.77$. The in-plane response reaches a local minimum near $\rho=0$ and increases thereafter until reaching the large downward jump in both $a_{3}$ and $a_{2}$ at $\rho \approx 13.85$. This downward jump is delayed until $\rho \approx 15.00$ when the excitation level is increased by $5 \%$ as shown by the position of $f_{t}$ in the 


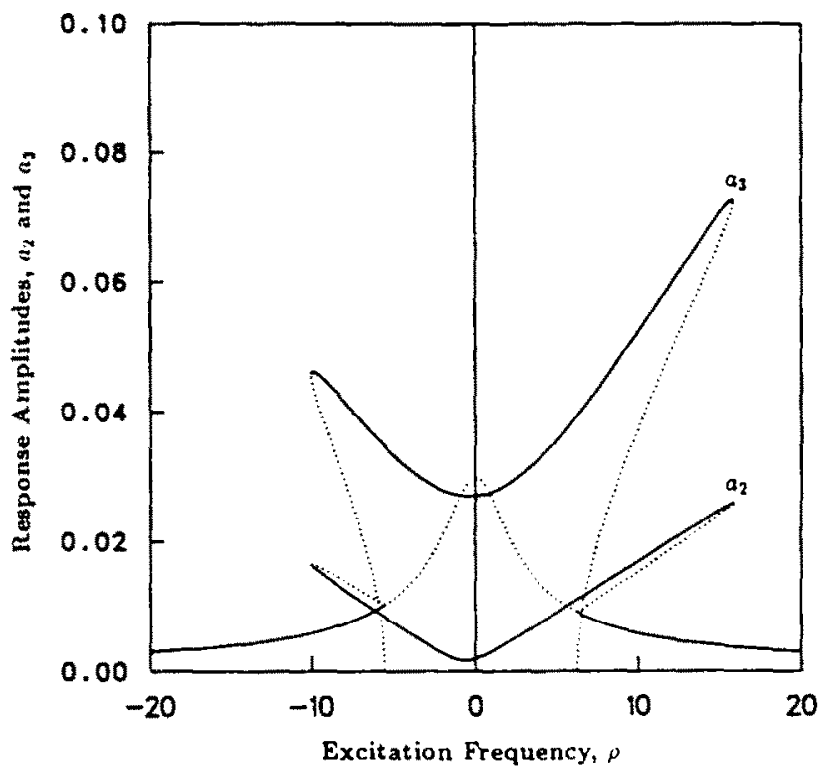

Fig. 6. Amplitude-frequency plot for case of vanishing internal detuning $(\sigma=0)$. Model parameters given in Table 1 and $f=2.0 \times 10^{-3} .(-)$ stable solutions; $(\cdots)$ unstable solutions.

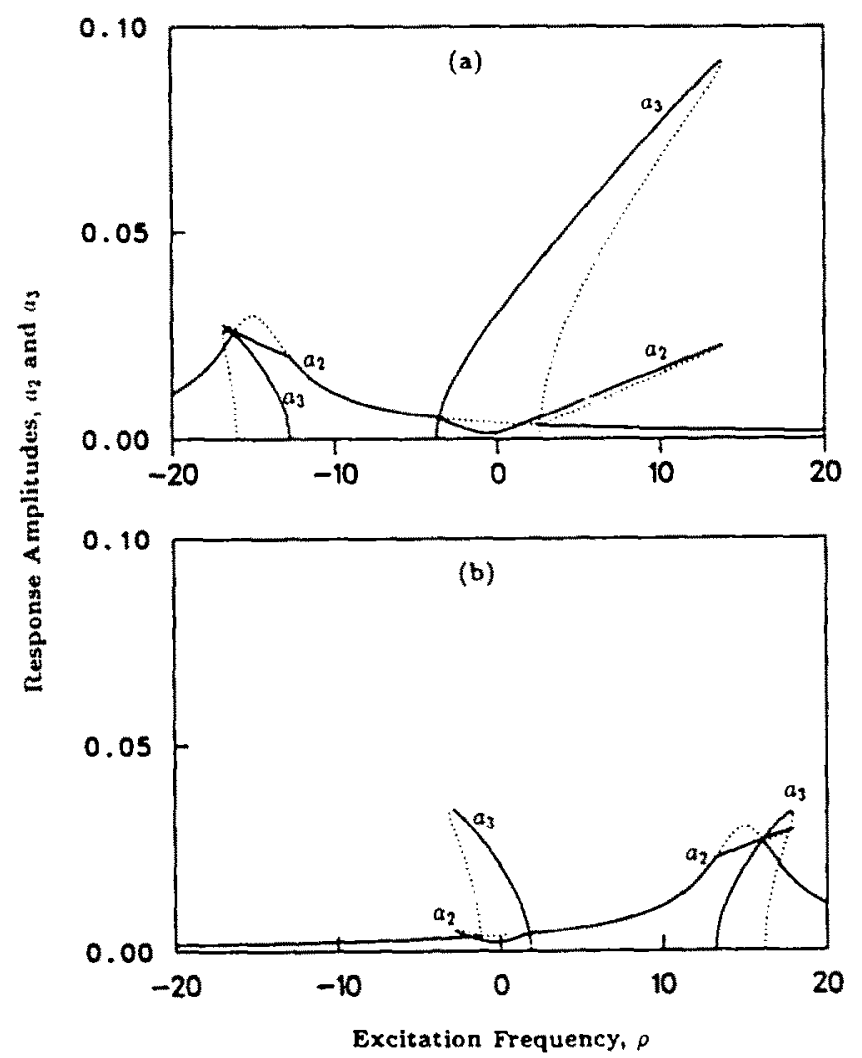

Fig. 7. Amplitude-(requency plots for cases of non-vanishing internal detuning. Model parameters given in Table $l$ and $f=2.0 \times 10^{-3}$. (a) $\sigma=15$; (b) $\sigma=-15$. (-) stable solutions; $(\cdots)$ ) unstable solutions.

amplitude-forcing plot of Fig. 5a. Consequently, the non-planar response depicted in Fig. 5a is driven primarily by the parametric resonance.

Overall, the positive internal detuning greatly enhances the non-planar response near the parametric resonance. By contrast, the non-planar response at the parametric resonance is 
greatly reduced in the case of significant negative internal detuning $(\rho=-15)$ as shown in Fig. $7 \mathrm{~b}$. In this case, the external resonance produces slightly larger non-planar response and lies to the right of the parametric resonance. Again, jumps occur at the extremes of the non-planar response regions $(\rho \approx 3.00,17.96)$ and the non-planar response is continuous with the (stable) planar response at the boundary of the intermediate region $(1.82<\rho<13.20)$. Finally, note that the non-planar response of Fig. $4 b(\sigma=-15, \rho=15)$ is mainly driven by the external resonance shown in Fig. 7b, while the non-planar response of Fig. $5 b(\sigma=-15, \rho=-15)$ is mainly driven by the parametric resonance.

As illustrated in the above results, the planar response loses stability for model parameter values that lead to bifurcations of the singular points determined by equations (41)-(44). Analysis of these equations leads to a closed-form expression for the bifurcation condition which governs the stability limit for planar response. Combining equations (41) and (42) provides

$$
a_{3}^{2}\left[\omega_{3}^{2}\left(\frac{1}{4} \rho^{2}+\mu_{3}^{2}\right)-\frac{1}{16} x_{1}^{2} a_{2}^{2}+\frac{1}{4} x_{1} \int p_{3} a_{2} \cos \left(\gamma_{2}-\gamma_{3}\right)-\frac{1}{4} f^{2} p_{3}^{2}\right]=0 .
$$

At the bifurcation point, both $a_{3}$ and the factor in brackets above vanish and $a_{2}$ is given by equation (45). Under these conditions, substitution of equations (43) and (45) into the factor in brackets results in the bifurcation condition expressed in terms of the model parameters:

$$
\omega_{3}^{2}\left(\frac{1}{4} \rho^{2}+\mu_{3}^{2}\right)=f_{b}^{2}\left\{\frac{1}{4} p_{3}^{2}+\frac{f_{2}^{2}}{4 \omega_{2}^{2}\left[\mu_{2}^{2}+(\rho+\sigma)^{2}\right]}\left[\frac{1}{16} \alpha_{1}^{2}+\frac{\alpha_{1} \omega_{2} p_{3}(\rho+\sigma)}{2 f_{2}}\right]\right\} .
$$

Here, $f_{b}$ again denotes the support motion amplitude at the bifurcation.

Figure 8 illustrates the bifurcation condition in the parameter plane defined by the support motion amplitude $f$ and frequency $\rho$ for the suspended cable example of Table 1 . Three sets of curves are shown corresponding to three values of internal detuning. In all cases, stable planar response occurs only for values of $(f, \rho)$ lying below the corresponding bifurcation curve defining $f_{b}$. The bifurcation curves exhibit local minima at values of $\rho$ that locate the positions of principal parametric resonance $(\rho=0)$ and primary external resonance $(\rho+\sigma=0)$. Thus, the curve corresponding to $\sigma=15(\sigma=-15)$ possesses two local minima at $\rho=0$ and $\rho=-15(\rho=15)$. These two resonances occur simultaneously at $\rho=0$ for the perfectly tuned cable $\sigma=0$ and lead to an order of magnitude reduction in $f_{b}$ over the cases $\sigma= \pm 15$ for $\rho=0$. Thus, the two-to-one internal resonance is responsible for the large reduction in the planar stability region near conditions of principal parametric resonance. Note also that, for the perfectly tuned cable, bifurcations for a prescribed value

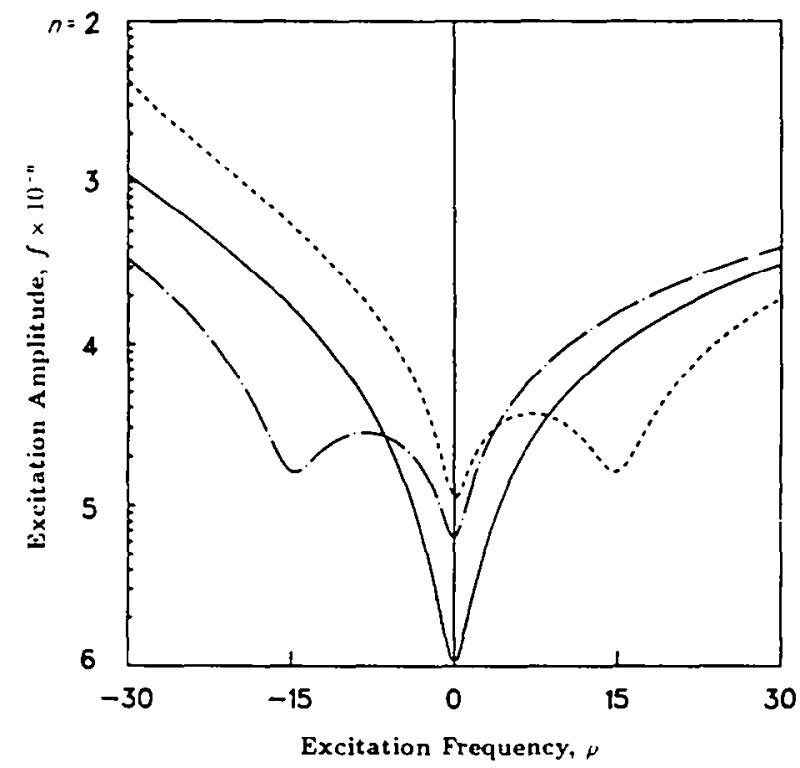

Fig. 8. Bifurcation condition in the excitation amplitude/frequency plane. Curves give $f_{0}$ for example of Table $1 .(-) \sigma=0,(-\cdot) \sigma=15 ;(-\cdots) \sigma=-15$. 
of $f_{b}$ occur at two values of $\rho$ at either side of the combined parametric/external resonance; compare with Fig. 6 using $f_{b}=2 \times 10^{-5}$. By contrast, up to four values of $\rho$ are associated with a prescribed value of $f_{b}$ in the cases where the parametric and external resonances are separated $\left(\sigma= \pm 15\right.$ ); compare with Fig. 7 using $f_{b}=2 \times 10^{-3}$.

\section{PRELIMINARY EXPERIMENTAL RESULTS}

As described in the Introduction, the non-linear response of a suspended cable under parametric excitation was first observed in a series of preliminary experimental tests. These tests were conducted using the cable test stand and instrumentation illustrated in Fig. 9.

The cable used in the experiment is a small-diameter, woven nylon cord that is suspended between two supports at the same elevation. The horizontal position of the right support of the cable is adjusted to achieve a sag to span ratio corresponding to a cable parameter approximately within the first crossover region $(\lambda / \pi \approx 2)$ shown in Fig. 1 . The left end of the cable is attached to a voice-coil shaker vibration exciter whose axis is aligned with cable's tangent. The shaker is driven by the sinusoidal output of a vibration exciter controller having controllable frequency and gain. A linear potentiometer fastened to the shaker provides direct measurement of the support motion amplitude. The response of the cable is measured using a special two-axis optical displacement probe developed in the laboratory. The probe, which follows from a previous design [28], utilizes a pair of linear diode arrays to measure the position of the cable cross-section in the normal $\left(U_{2} \times U_{3}\right)$ plane. Along both axes, the cable is illuminated by an external light source and the resulting shadows are focused onto the diode arrays. Each diode array consists of 256 photo diodes on $25 \mu \mathrm{m}$ centers and, at any time, only a few diodes are covered by the cable shadow. A circuit was designed to continuously scan the diode array and to store the location of the covered diodes. Consequently, the location of the cable cross-section (shadow) can be measured and at scanning rates adjustable up to $5 \mathrm{kHz}$. The circuit outputs are recorded by a digital computer data aquisition system and are later calibrated. This probe makes non-contacting measurements of the large amplitude cable response and is capable of detecting the position of the cable cross-section (4.5 mm in diameter) in a $16 \times 16 \mathrm{~cm}^{2}$ area to within $0.7 \mathrm{~mm}$.

To begin the experiment, the free response of the cable to initial conditions was measured to estimate the natural frequencies of the fundamental out-of-plane and symmetric in-plane modes as in [28]. These estimates indicated that $\omega_{2} \approx 2 \omega_{3}$ with small (positive) detuning $\varepsilon \sigma \approx 0.4$ and confirmed that the cable was within the region of the first crossover. In the remainder of the experiment, the support motion frequency was held constant and the exciter gain was adjusted in a quasi-static manner. After each gain change, the cable response was allowed to return to steady state and sampled signals from the probe were recorded and the support motion amplitude was noted. The support motion frequency was

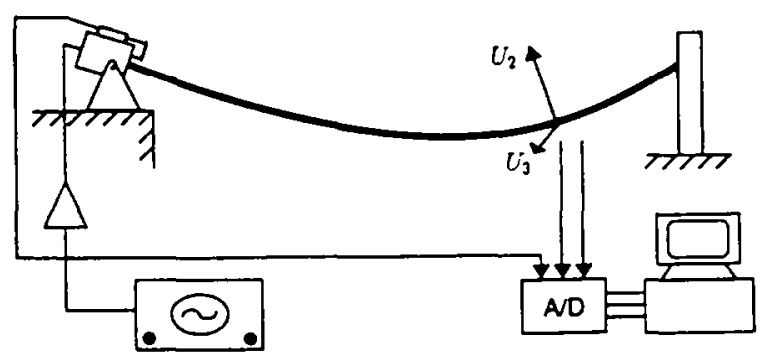

Fig. 9. Schematic of cable test stand and instrumentation. Cable: $4.5 \mathrm{~mm}$ diameter woven nylon cord, $\rho=12.0 \mathrm{~g} / \mathrm{m}, E A=1900 \mathrm{~N}$. Two-axis optical displacement probe: measures position of cable cross-section in the normal plane defined by the normal $U_{2}$ and binormal $U_{3}$ displacements; range: $16 \times 16 \mathrm{~cm}^{2}$; accuracy: $0.7 \mathrm{~mm}$; frequency response: $\leqslant 5 \mathrm{kHz}$. Shaker: voice-coil shaker generates small, tangential oscillations of left support which are measured by attached potentiometer. Signal generator: drives shaker with controllable frequency and gain; frequency resolution: $0.001 \mathrm{~Hz}$. Data acquisition unit: microcomputer equipped with an $A, D$ board used to sample, store and reduce data. In current test: cable length $L=3.615 \mathrm{~m}$; probe placed $1.16 \mathrm{~m}$ from shaker; frequency constant $3.300 \mathrm{~Hz}$. 

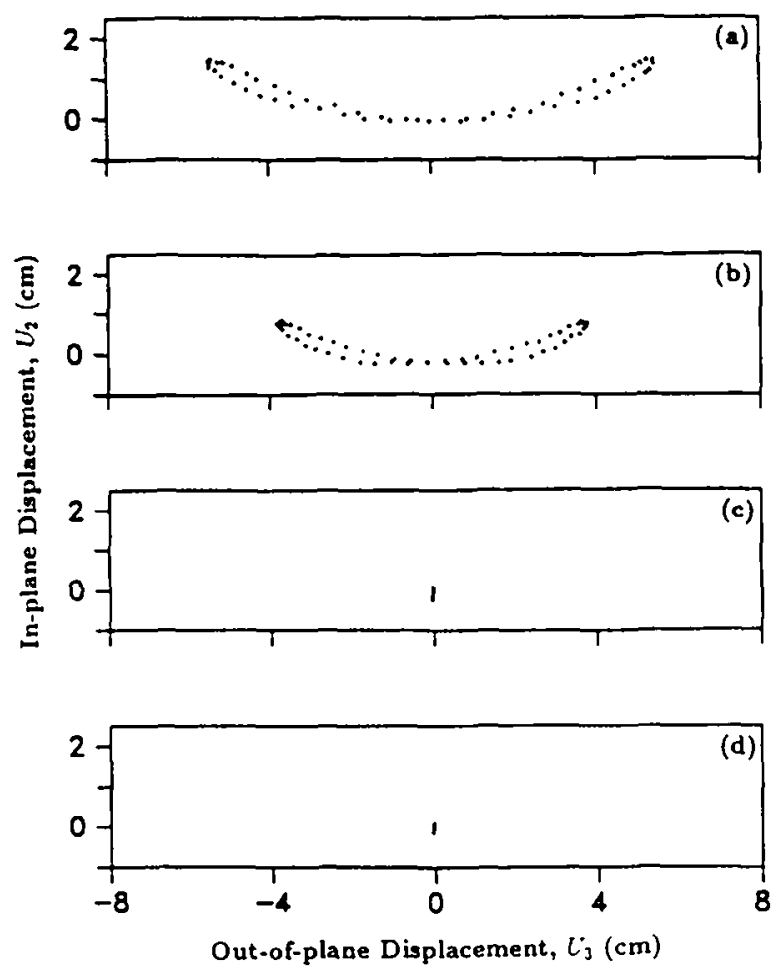

Fig. 10. The sequence (a)-(d) shows the periodic orbits generated by slowly decreasing the excitation amplitude $F$ from maximum value of $F=0.85 \mathrm{~mm}$; refer to Fig. $2 \mathrm{~d}$. (a) $F=0.51 \mathrm{~mm}$ $\left(f=14.1 \times 10^{-5}\right) ; \quad$ (b) $F=0.22 \mathrm{~mm} \quad\left(f=5.96 \times 10^{-3}\right) ; \quad$ (c) $F=0.20 \mathrm{~mm} \quad\left(f=5.50 \times 10^{-3}\right)$; (d) $F=0.14 \mathrm{~mm}\left(f=3.82 \times 10^{-5}\right)$.

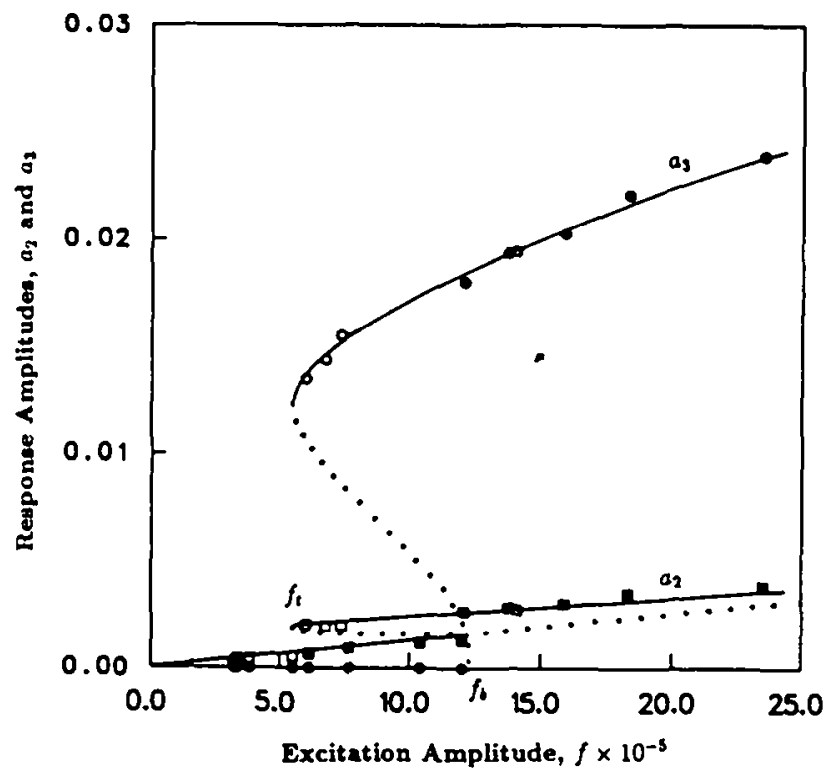

Fig. 11. Experimental results for amplitude-forcing plot. Non-dimensional steady-state amplitudes of in-plane $\left(\square \square a_{2}\right)$ and out-of-plane $\left(\odot \bigcirc a_{3}\right)$ motions are plotted vs non-dimensional support motion amplitude $f$. Solid (open) symbols denote data collected while increasing (decreasing) $f$. Curves are sketched through data.

selected to excite the principal parametric resonance of the out-of-plane mode and the (positive) parametric detuning was estimated to be $\varepsilon \rho \approx 0.8$.

Figure 2 shows a sequence of periodic responses that are representative of those measured while slowly increasing the support motion amplitude $f$. For the low-amplitude cases 
of Fig. $2 \mathrm{a}$ and $\mathrm{b}$, the response is planar and grows linearly with increasing $f$. Increasing $f$ beyond a critical value $f_{b} \approx 12.0 \times 10^{-3}$, leads to large non-planar response. In the periodic orbit shown in Fig. $2 \mathrm{c}$, the in-plane motion completes two cycles for every cycle of the out-of-plane motion. This clear two-to-one resonant response is further enhanced at larger values of $f$ as shown in Fig. $2 d$. In this extreme case, a support motion amplitude of only $0.85 \mathrm{~mm}$ leads to large in-plane and out-of-plane responses with amplitudes 1.08 and $6.73 \mathrm{~cm}$, respectively. Slowly decreasing the amplitude leads to a gradual reduction in the non-planar response until it suddenly collapses to pure in-plane response below a critical value $f_{t} \approx 5.7 \times 10^{-5}$; refer to Fig. 10 . Note also that the orbit shown in Fig. 10a $\left(f=14.1 \times 10^{-5}\right)$ for decreasing $f$ reproduces that in Fig. $2 \mathrm{c}\left(f=13.8 \times 10^{-5}\right)$ for increasing $f$.

The results from the entire test are summarized in the amplitude-forcing plot of Fig. 11. The solid (open) symbols represent response amplitudes measured for increasing (decreasing) $f$. The range $0 \leq f \leq f_{b}$ defines the region of (stable) planar response which partly overlaps the region for (stable) non-planar response $f_{t} \leq f$ due to non-vanishing external detuning. Note that the curves sketched through the data in Fig. 11 are in good qualitative agreement with those of Fig. 5a for the case of positive internal and parametric detuning. The calculated results in Fig. 5a are, however, carried out for values of $f$ well beyond those indicated in Fig. 11.

\section{SUMMARY AND CONCLUSIONS}

A theoretical model is derived which describes the three-dimensional, non-linear response of a suspended elastic cable to tangential oscillations of one support. The model shows that small support oscillations lead to parametric excitation of out-of-plane motion and simultaneous parametric and external excitation of in-plane motion. A two-degree-of-freedom approximation of the model is employed to examine a class of in-plane/out-of-plane motions that are coupled through the quadratic non-linearities created by non-zero equilibrium cable curvature.

A first-order perturbation analysis is utilized to determine the existence and stability of planar and non-planar periodic motions. The analysis focuses on the periodic motions that arise under conditions of principal parametric resonance of the fundamental out-of-plane mode for a suspended cable near the first crossover region. In this region, a two-to-one internal resonance exists between the fundamental out-of-plane mode and the first symmetric in-plane mode. For such cases, support motions producing principal parametric resonance of the out-of-plane mode also produce primary external resonance of the in-plane mode and the separation of these two resonances is proportional to the magnitude of the internal detuning. Furthermore, the principal parametric resonance disrupts the saturation phenomenon that would otherwise occur in the presence of primary external resonance alone. A bifurcation condition governing planar stability is derived and results indicate that the two-to-one internal resonance greatly reduces planar stability and enhances non-planar response. Such tuned resonant behavior is clearly observed in experimental measurements of cable response which are also in good qualitative agreement with theoretical predictions.

Acknowledgements - The author gratefully acknowledges the support of this research endeavor provided by the U.S. Office of Naval Research Young Investigator Program through Grant No. N00014-89-J-3159. The author is also grateful to $\mathrm{Mr}$ Christopher Lee for his efforts in developing the experimental apparatus.

\section{REFERENCES}

1. H. M. Irvine, Cable Structures. MIT Press, Cambridge, MA (1981).

2. M. S. Triantafyllou, Linear dynamics of cables and chains. Shock Vib. Dig. 16, 9 (1984).

3. M. S. Triantafyllou, Dynamics of cables and chains. Shock Vib. Dig. 19, 3 (1987).

4. H. M. Irvine and T. K. Caughey, The linear theory of free vibrations of a suspended cable. Proc. R. Soc. Lond. A341, 299 (1974).

5. P. Hagedorn and B. Schafer, On non-linear free vibrations of an elastic cable. Int. J. Non-Linear Mech. 15, 333 (1980).

6. G. Rega. F. Vestroni and F. Benedettini, Parametric analysis of large amplitude free vibrations of a suspended cable. Int. J. Solids Struct. 20, 95 (1984). 
7. A. Luongo, G. Rega and F. Vestroni, Planar non-linear free vibrations of an elastic cable. Int. J. Non-Linear Mech. 19, 39 (1984).

8. A. Luongo, G. Rega and F. Vestroni, Monofrequent oscillations of a non-linear model of a suspended cable. J. Sound Vib. 82, 247 (1982).

9. F. Benedettini, G. Rega and F. Vestroni, Modal coupling in the free nonplanar finite motion of an elastic cable. Meccanica 21, 38 (1986).

10. A. Luongo, G. Rega and F. Vestroni, Free oscillations of a nonlinear simple model of a suspended cable. Proc. Recent Adv. Struct. Dyn., Institute of Sound and Vibration Research, Vol. 2, p. 597 (Edited by M. Petyt) (1980).

11. K. Takahashi and Y. Konishi. Non-linear vibrations of cables in three dimensions. Part I: Non-linear free vibrations. J. Sound Vib. 118, 69 (1987).

12. F. Benedettini and G. Rega, Non-linear dynamics of an elastic cable under planar excitation. Int. $J$. Non-Linear Mech. 22, 497 (1987).

13. F. Benedettini and G. Rega, Planar non-linear oscillations of elastic cables under superharmonic resonance conditions. J. Sound Vib. 132, 353 (1989).

14. F. Benedettini and G. Rega, Planar non-linear oscillations of elastic cables under subharmonic resonance conditions. J. Sound Vib. 132, 367 (1989).

15. S. I. Al-Noury and S. A. Ali, Large-amplitude vibrations of parabolic cables. J. Sound Vib. 101, 451 (1985).

16. K. Takahashi and $Y$. Konishi, Non-linear vibrations of cables in three dimensions. Part II: Out-of-plane vibrations under in-plane sinusoidal time-varying load. J. Sound Vib. 118, 85 (1987).

17. H. Yamaguchi, T. Miyata and M. Ito, A behavior on nonlinear dynamic response of cable systems. Proc. 24th Symp. Struct. Engng p. 55 (1978).

18. A. H. Nayfeh and B. Balachandran, Modal interactions in dynamical and structural systems. Appl. Mech. Rev. 42, Part 2, 175 (1989).

19. P. R. Sethna, Vibrations of dynamical systems with quadratic nonlinearities. ASME J. appl. Mech. 32, 576 (1965).

20. J. W. Miles, Resonant forced motion of two quadratically coupled oscillators. Physica D 13, 247 (1984).

21. A. H. Nayfeh, The response of two-degree-of-freedom systems with quadratic nonlinearities to a parametric excitation. J. Sound Vib. 88, 547 (1983).

22. J. W. Miles, Parametric excitation of an internally resonant double pendulum. J. appl. Math. Phys. (Z.A.MP) 36 . 337 (1985)

23. A. G. Haddow, A. D. S. Barr and D. T. Mook, Theoretical and experimental study of modal interaction in a two-degree-of-freedom structure. J. Sound Vib. 97, 451 (1984).

24. A. H. Nayfeh and L. D. Zavodney, Experimental observation of amplitude- and phase-modulated responses of two internally coupled oscillators to a harmonic excitation. ASME J. appl. Mech. 55, 706 (1988).

25. $\mathrm{H}$. Troger and C. S. Hsu, Response of a nonlinear system under combined parametric and forcing excitation. ASME J. appl. Mech. 44, 179 (1977).

26. N. C. Perkins and C. D. Mote Jr, Three dimensional vibration of travelling elastic cables. J. Sound Vib. 114, 325 (1987).

27. A. H. Nayfeh and D. T. Mook, Nonlinear Oscillations. Wiley Interscience, New York (1979).

28. N. C. Perkins and C. D. Mote Jr, Theoretical and experimental stability of two translating cable equilibria. J. Sound Vib. 128, 397 (1989).

\section{APPENDIX: DISCRETE MODEL}

The parameters appearing in the discrete model in equations (28) and (29) are determined through application of Galerkin's method using the in-plane and out-of-plane vibration mode shapes associated with free, linear response. The (normalized) out-of-plane modes correspond to those of a taut string and are given by

with natural frequencies

$$
\begin{gathered}
\theta_{3 j}(s)=\sqrt{2} \sin j \pi s \\
\omega_{3}=j \pi v_{t}, \quad j=1,2,3, \ldots .
\end{gathered}
$$

The in-plane modes are those associated with an elastic cable [4] and are distinguished as being either symmetric or anti-symmetric with respect to the mid-span. The (normalized) antisymmetric in-plane modes,

$$
\theta_{2 i}(s)=\sqrt{2} \sin i \pi s, \quad i=2,4,6, \ldots \text { (antisymmetric) }
$$

with natural frequencies

$$
\omega_{2}=i \pi v_{t}, \quad i=2,4,6, \ldots,
$$

induce no dynamic tension (no first-order cable stretching) and are identical to those of a taut string. The (normalized) symmetric in-plane modes,

$$
\theta_{2 i}(s)=\frac{2 Q}{\cos \left(\omega_{2} / 2 v_{1}\right)}\left[\sin \frac{\omega_{2} s}{2 v_{1}} \sin \frac{\omega_{2}(s-1)}{2 v_{1}}\right] \quad i=1,3,5, \ldots \text { (symmetric) }
$$

induce (first-order) dynamic tension and have natural frequencies $\omega_{2}$ determined by the roots of the characteristic equation,

In equation (53),

$$
\tan \frac{\omega_{2}}{2 v_{t}}-\frac{1}{2}\left[\frac{\omega_{2}}{v_{1}}-\frac{\left(\omega_{2} / v_{1}\right)^{3}}{\lambda^{2}}\right]=0
$$

$$
\lambda^{2}=\frac{v_{l}^{2}}{\left(v_{t}^{2}\right)^{3}}
$$


is the cable parameter introduced by Irvine and Caughey [4] and in equation (52)

$$
Q=\sqrt{\frac{\left(\omega_{2} / v_{t}\right)\left[1+\cos \left(\omega_{2} / v_{t}\right)\right]}{\left(\omega_{2} / v_{r}\right)\left[2+\cos \left(\omega_{2} / v_{t}\right)-3 \sin \left(\omega_{2} / c_{t}\right)\right]}}
$$

is a normalization constant.

In equations (28) and (29), the coefficients of the non-linear terms $x_{1}-\beta_{4}$, the parametric excitation terms $\hat{\beta}_{2}$ and $\hat{p}_{3}$, and the external excitation term $\hat{f}_{2}$ are determined after first evaluating the quantities,

and

$$
\bar{\theta}_{2}=\int_{0}^{1} \theta_{2 i}(\eta) \mathrm{d} \eta=\left\{\begin{array}{cl}
0 & \text { antisymmetric } \theta_{2 i}(s) \\
Q\left[1+\frac{2 v_{\mathrm{r}}\left[\cos \left(\omega_{2} / v_{\mathrm{t}}\right)-1\right]}{\omega_{2} \sin \left(\omega_{2} / v_{\mathrm{r}}\right)}\right] & \text { symmetric } \theta_{2 i}(s)
\end{array}\right.
$$

$$
r^{2}=\omega_{2}^{2}-\frac{v_{l}^{2}}{\left(t_{t}^{2}\right)^{2}} \theta_{2}^{2} \text {. }
$$

Then,

$$
\begin{aligned}
& x_{1}=\frac{v_{t}^{2}}{\left(v_{t}^{2}\right)^{2}} \omega_{3}^{2} \bar{\theta}_{2}, \quad x_{2}=\frac{1}{2} x_{1}, \quad x_{3}=\frac{3}{2} \frac{v_{l}^{2}}{\left(v_{t}^{2}\right)^{2}} r^{2} \bar{\theta}_{2} \\
& \beta_{1}=-\frac{1}{2} \frac{v_{1}^{2}}{\left(v_{t}^{2}\right)^{2}} \omega_{3}^{3}, \quad \beta_{2}=\beta_{3}=-\frac{1}{2} \frac{v_{l}^{2}}{\left(v_{t}^{2}\right)^{2}} r^{2} \omega_{3}^{2} . \quad \beta_{d}=-\frac{1}{2} \frac{v_{t}^{2}}{\left(v_{t}^{2}\right)^{2}} r^{+} \\
& \hat{p}_{2}=-\frac{1}{2} \frac{v_{l}^{2}}{2} r_{t}^{2}, \quad \hat{p}_{3}=-\frac{1}{2} \frac{v_{l}^{2}}{2 v_{t}^{2}} \omega_{3}^{2}, \quad \hat{f}_{2}=-\frac{v_{l}^{2}}{v_{t}^{2}} \bar{\theta}_{2} .
\end{aligned}
$$

Notice that for antisymmetric in-plane modes, $\theta_{2}=0$, and the quadratic non-linearities and external excitation all vanish $\left(x_{1}=x_{2}=x_{3}=\hat{f}_{2}=0\right)$. 